Gut, 1966, 7, 666

\title{
Recurrent gastric carcinoma treated by the Geiger probe technique
}

\author{
D. J. MARTIN \\ From the Department of Radiotherapy, United Bristol Hospitals
}

EDITORIAL COMMENT Conventional radiotherapy or chemotherapy has little success in the treatment of adenocarcinoma of the stomach. It is suggested that if radiotherapy can be given at times of tumour mitotic activity then greater success might be achieved. In this case a pattern of tumour activity was induced by Methotrexate and radiotherapy given during peaks of activity as judged by the Geiger probe technique. A single case is recorded though, naturally, conclusions cannot be drawn from an isolated success.

It is generally considered that the treatment of carcinoma of the stomach by radiotherapy is of little value (Paterson, 1963), and, although surgery is the treatment of choice for those lesions which are operable, in most cases it has nothing to offer in the treatment of recurrences (Pöntinen, 1963).

From 148 evaluated cases treated by radiotherapy alone Holdfelder (1938) had three five-year survivals, the average duration of life being 10.6 months. Kettunen and Larmi (1964) found a beneficial effect of convergent beam therapy for the first four months but not later.

Chemotherapy has also produced equally poor results, the average response being five to nine months (Langdon, Ottoman, Rochlin, and Smart, 1963; Hurley, Ellison, and Carey, 1961).

The following case is one in which the Geiger probe technique has been used in association with radiotherapy (Hale, 1961; Bullen, Freundlich, Hale, Marshall, and Tudway, 1963).

\section{CASE REPORT}

A 42-year-old lorry driver was admitted to Southmead Hospital on 12 April 1961 with an 18-month history of nausea, vomiting, eructation, and weight loss. He had experienced epigastric fullness after meals which was relieved by vomiting and had lost $10 \mathrm{lb}$. in the previous year. Examination revealed no abnormalities except a succussion splash, there being no palpable abdominal masses. Two barium meals at an interval of nine days showed pyloric stenosis with considerable delay in gastric emptying and a filling defect in the antrum on the greater curve. A laparotomy was carried out on 2 May 1961 when a large ulcer cancer was seen in the pyloric antrum. No hepatic metastases or enlarged lymph nodes were noted, and a wide Billroth 1 gastrectomy was performed.
Histology showed a well-differentiated adenocarcinoma involving the full thickness of the stomach wall. An otherwise uneventful post-operative period was interrupted by a deep vein thrombosis which was treated by anticoagulants, the patient being discharged home on 21 May 1961.

On 16 April 1962 the patient was referred for radiotherapy, having a history of a lump increasing in size at the lower end of the laparotomy scar for three months. A biopsy of the mass confirmed that it was a secondary deposit of the gastric tumour and a Geiger probe was buried in the mass. Following the administration of 750 microcuries of ${ }^{32} \mathrm{P}$ peaks of tumour activity occurring at nearly regular intervals were induced by means of Methotrexate ( $2.5 \mathrm{mg}$. b.d. for four days). Treatment with deep $x$ rays $(220 \mathrm{KV}$ H.V.L. $1.5 \mathrm{~mm}$. Cu) was given on the rise of the peaks, a total skin dose of 4,400 rads being given in 15 days from 12 treatments. At the time of discharge on 17 May 1962, the mass was much smaller and the patient was quite well. By September 1962 the mass had become impalpable.

The patient remained well until 28 September 1964 when he was readmitted, complaining that he could no longer eat large pieces of food because they caused vomiting. There was also epigastric pain after meals and he had lost $10 \mathrm{lb}$. in weight in six weeks. At this time two more hard masses had become palpable in the scar and again biopsy confirmed a well-differentiated adenocarcinoma. Similar treatment as on the previous occasion was given, using ortho-voltage and the Geiger probe technique, 2,700 rads being delivered to the centre of the tumour in 15 treatments over 32 days. It was noted that the regular peaks of activity in the tumour induced on the previous admission were still present although no further Methotrexate was given. Also, after only 1,000 rads had been given, the tumour was scarcely palpable, finally disappearing by December 1964.

On 1 March 1965 the patient was again admitted because of epigastric discomfort, occasional vomiting, 
diarrhoea, and a loss of weight of 1 stone in two months. A hard irregular mass was palpable in the suprapubic region. A barium meal and follow-through demonstrated no abnormality of the stomach remnant or of the small bowel. Laparotomy showed the mass to be in the anterior abdominal wall just deep to the rectus muscles. As before Geiger probes were inserted and again spontaneous cycles were seen. During the following 25 days, 2,530 rads were given to the centre of the tumour in 11 treatments. By 18 March the diarrhoea and vomiting had cleared up and the mass had become softer. When last seen on 3 May 1965 the mass had almost gone and the patient was feeling better.

\section{DISCUSSION}

The above case is taken from a series at present forming part of a research project in which many different types of malignant tumour, normally considered to be radio-resistant or having been subjected to a conventional course of radiotherapy without response, are treated by the technique of controlled radio-therapy. Because of similar responses seen in these patients it is considered that the favourable result in the case presented may be due to the fact that radio-therapy was given at times when the tumour was most radio-sensitive. The many cases in which conventional radiotherapy or chemotherapy have been used with little success, it is felt, act as controls to the present work. It is interesting that the pattern of tumour activity induced initially has been maintained although no further Methotrexate was given.

\section{SUMMARY}

A case of gastric carcinoma is described in which the original lesion was resected and subsequently three recurrences were successfully treated by means of radiotherapy, controlled by the Geiger probe technique. For the greater part of four years the patient has remained at work and in good health.

I wish to thank Dr. R. C. Tudway, Dr. J. M. Naish, and Mr. W. M. Capper for permission to report this case under their care, and Dr. B. T. Hale and Dr. A. E. A. Read for advice and criticism.

\section{REFERENCES}

Bullen, M. A., Freundlich, H. F., Hale, B. T., Marshall, D. H., and Tudway, R. C. (1963). The activity of malignant tumours and response to therapeutic agents, studied by continuous records of radioactive phosphorus uptake. Postgrad. med. J., 39, 265-277.

Hale, B. T. (1961). A technique for studying human tumour growth in vivo. Lancet, 2, 345-347.

Holdfelder, H. (1938). 'Rontgentherapy in Diesase of Gastro-intestinal tract including its glands'. In Clinical Rontgen Therapy, edited by E. A. Pohle, Kimpton, London.

Hurley, J. D., Ellison, E. H., and Carey, L. C. (1961). Treatment of advanced cancer of the gastrointestinal tract with antitumour agents. Gastroenterology, 41, 557-562.

Kettunen, K., and Larmi, T. (1964). Convergent beam therapy following gastrectomy for gastric cancer. Acta chir. scand., 127, 138-140.

Langdon, E. A., Ottoman, R. E., Rochlin, D. B., and Smart, C. R. (1963). Early results of combined radiation and chemotherapy in treatment of malignant tumours. Radiology, 81, 1008-1013.

Paterson, R. (1963). The Treatment of Malignant Disease by Radiotherapy. Arnold, London.

Pöntinen, P. J. (1963). Results of Treatment in Gastric Carcinoma. Ann. Chir. Gynaec. Fenn., 52, suppl. 127. 